\title{
Keeve Information Identification Technique for Spectrum Sharing Based on Geo- location, Time and Frequency for Cognitive Radio Networks
}

\author{
Amrit Mukherje* and Amlan Datta
}

School of Electronics Engineering KIIT University, India

Received 8 June 2016; Accepted 2 July 2016

\begin{abstract}
The Letter investigates the spectrum sharing based operation for Cognitive Radio (CR) users and network based on the observation of pre-information of a location or the nature of the $\mathrm{CR}$ users. A new empirical technique i.e. Keeve Information Identification Technique (KIIT) is used to estimate the information based sharing for a mobile CR with respect to time and frequency band availability. We use the geo-space or location information in sharing to determine the likelihood of getting shared by networks at two different location and time. The novel aspect of the spectrum sharing on the basis of three basic parameters is designed. These are the geo-space, time and the networks (frequency bands) and a Cognitive Radio (CR) transceiver is considered as moving and entering any location searching for spectrum sharing. The different parameters for calculation are taken as uninformed and informed CR, and geo-space based information availability. The likelihood function is analyzed based on energy detection method where the Power Spectrum Density of the channel is considered as parameters for informed and informed CR independent of time and location. The mobility of the CR user generates the likelihood of spectrum sharing with the available networks. The overall operations are assumed to be in form of Poisson process.
\end{abstract}

Keywords: Keeve Information Identification Technique, Spectrum Sharing, Wireless Communication, Cognitive Radio Network

\section{Introduction}

Broadly the approach of efficient spectrum usage through cognitive radio is being elaborated using the two proposed methods: i) dynamic spectrum access based on the spectrum sensing outputs [1,2] where the secondary or the CR user sense the channel and occupies only when the primary users or licensed users are not present and, ii) using spectrum sharing using Underlay or Overlay schemes [3,4] where the CR user coexist with primary users based on a separation gap in power levels without interfering the later. Further on architecture level sharing includes Centralized and Decentralized [5] where the spectrum allocation and access procedure will be governed by a central entity and for Decentralized it is governed on local basis. Previously a lot of research has been focused on different strategies for capacity enhancing of spectrum sharing based on optimal power allocation which maximizes the ergodic capacity using average interference and power constraints [6]. In our proposed model, we have proposed a sharing model, where the motion and different sharing parameters are considered based on Poisson process. Here, we have proposed the design based on the mobility of $\mathrm{CR}$ i.e geo-space information availability and Network's contribution towards sharing with CR for a continuous or instantaneous time

\footnotetext{
*E-mail address: amrit1460@gmail.com ISSN: 1791-2377 @ 2016 Eastern Macedonia and Thrace Institute of Technology. All rights reserved.
}

scale. In section 2, the proposed model has been explained and section 3 highlights the mathematical implementation of our algorithm based on likelihood of occurrence of sharing. In general, we have considered the Power Spectral Density (PSD) of a same channel at different positions and at different time. These makes the input parameters of the algorithms independent of each other and later the likelihood is found out using optimized set and minimum search algorithm. The proposed model Keeve Information Identification Technique (KIIT) has been described in section 2. The analysis of the model and the simulation results shows a valid likelihood of the sharing performance.

The model is based on the assumptions that the CR transceiver observes the sharing in a given area as it is on move and for a particular day as $\alpha$. When the location update takes place, the available networks not ready to share is with probability $\delta$. Informed CR enters a geographical area with probability $\mu$ and uninformed CR participating in sharing with a probability $1-\mu$. $\lambda$ denotes the uninformed sharing instantly while as $1-\lambda$ is the probability of uninformed sharing continuously between the CR and the Network. Assuming the present of an uninformed CR, who is waiting for the sharing availability with probability of sharing instantly is denoted as $\varepsilon s$ and no sharing as 1-Es. Uninformed CR sharing with network ready for sharing continuously and not sharing is given by $1-\varepsilon g$.

\section{Proposed Model KIIT}

In this model, we set different instant and continuous sharing modes with respect to time, and other cases of geo-space 
based dependability of sharing parameters. The motivation behind the model come from the word "KEEVE" which means a large bucket or tub. The tub is compared with a geo-space where the uninformed or informed CR transceiver enters into it. The corresponding behavior after entering the KEEVE is sharing with the network available in a continuous or instantaneous manner. The model focuses on the mobility of $\mathrm{CR}$ transceivers arrival to a particular location for continuous sharing, keeping the frequency allotment constant with respect to that geo-space. Our main aim is to calculate empirically the sharing attributes. Assuming single CR shares with available network in a single available channel for $\mathrm{i}=1, \ldots . ., \mathrm{I}$ time. For any single day and continuous time should be $\mathrm{i} C[0, \mathrm{~T}]$. The networks are ready to share with $\mathrm{CR}$ based on $\mathrm{CR}$ information. Prior to sharing operation, the study of the nature of geo-space database and network sharing of the region is mandatory. Location based information is very important with respect to a fixed day or time for initialization of sharing process. Assuming an independent movement of CR transceiver(s) and independent distribution of network as $\alpha$ and probability of ready to share as $(1-\delta)$ or not ready to share as $\delta$. After a single sharing process completed and before the next sharing process starts, the basic model setup is assumed to takes its default values.

Let $\left(V_{i}\right)_{i=1}^{I}$ be the variables giving the sharing parameters at the end of each sharing processes $i=1, \ldots ., I$. Assuming the geo-space location is available and the network presents are ready to share at time $\mathrm{i}$ with parameter value be $\tilde{\mathrm{V}} \mathrm{i}$ and similarly for not sharing case at time $\mathrm{i}$ as (Vi). For no sharing condition and for a particular time i, the variable is given by $\left(\mathrm{V}^{*} \mathrm{i}\right)$. Assuming the case that $(\bigvee i)<\left(V_{i}^{*}\right)<(\tilde{\mathrm{V}} \mathrm{i})$ [8]. Sharing is performed from both informed and uninformed CR and when an uninformed CR transceiver arrives are assumed to follow Poisson process. The uninformed $\mathrm{CR}$ and the uninformed network exist at $\varepsilon$ which is the rate of sharing per unit time. If the CR observes good sharing conditions, it may start its own process without being cared for other CR present in that geo-space. The rate of arrival of this uninformed $\mathrm{CR}$ is $\mu$. For every individual CR arrivals, all of these are assumed to be independent. The tree diagram shows the sharing model based on geo-space network behaviors and time.

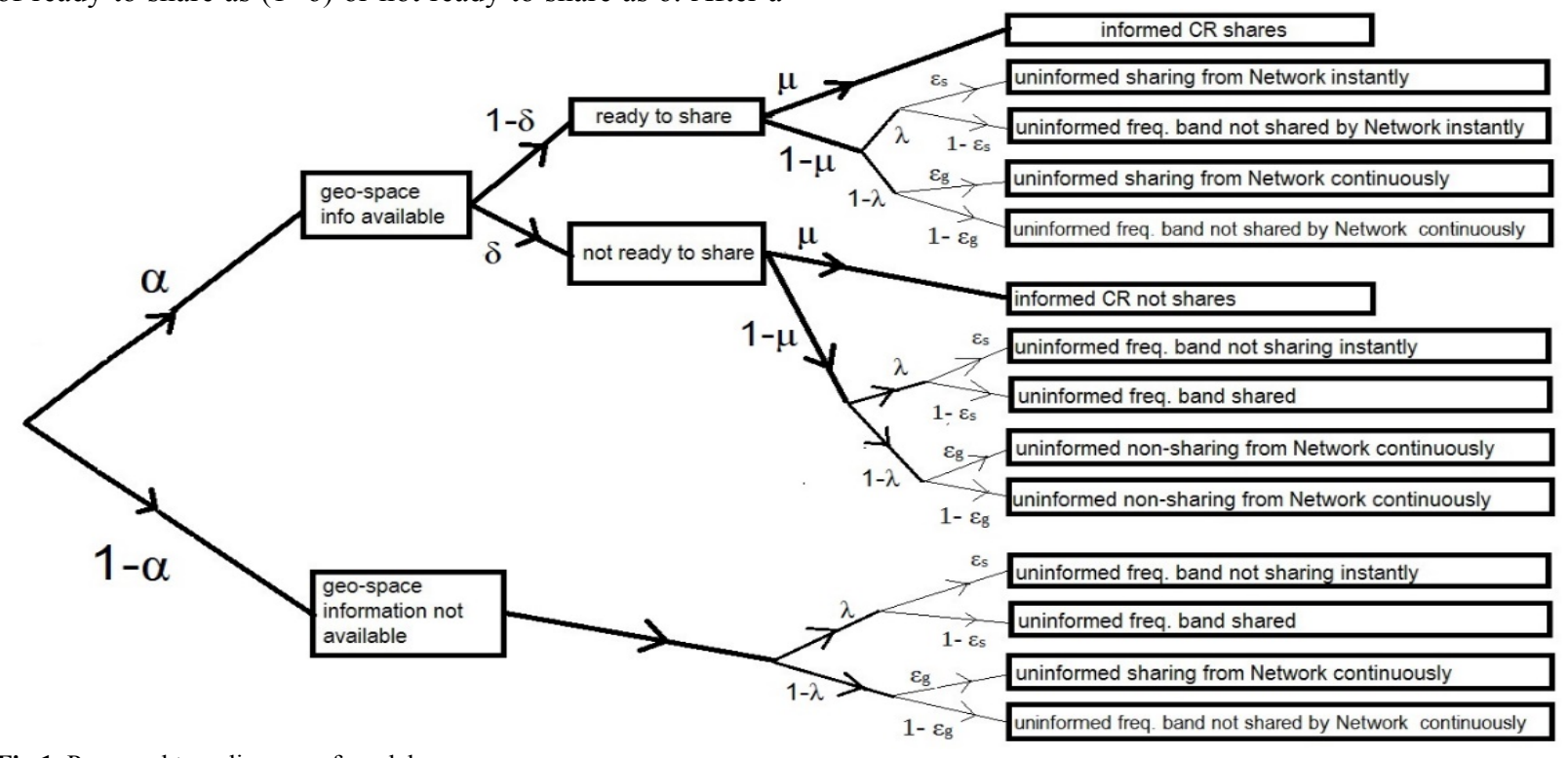

Fig 1. Proposed tree diagram of model

At the first node of the tree, which is based on geospace information whether available or not. For a geospace based information available information, and positive sharing conditions, the $\mathrm{CR}$ arrival rates are given by $\varepsilon^{+} \mu$ for continuous sharing and $\varepsilon$ for instantaneous based. Finally for non-sharing conditions, the uninformed CR arrives at arrival rate of $\varepsilon$ without or instantaneous sharing.

\section{Likelihood Function Analysis}

Defining the parameter vector $(\theta)=(\alpha, \delta, \varepsilon, \mu)$ where parameter $\alpha$ and parameter $\delta$ defines the conditional probability of sharing when Geo-space information available, geo-space information available but not sharing and geo-space information not available. The rest of the parameters represents informed and uninformed CR arrival rates. Assuming the proposed model follows one of the three Poisson processes for each time. The likelihood of observing any sharing with geo-space location available for a particular time $\mathrm{T}$ is given by:

$$
\begin{aligned}
& \operatorname{prob}(G, S, N \mid s=\text { share })=[\mu+(1-\mu) \lambda \varepsilon]^{G} \\
& *[(1-\mu) \lambda \varepsilon]^{S}[2(1-\mu)(1-\varepsilon) \lambda]^{N}
\end{aligned}
$$

Similarly when geo-space information is not available, the likelihood of sharing and non-sharing is given by:

$$
\begin{aligned}
& \operatorname{prob}(G, S, N \mid s=\text { notshare })= \\
& {[\mu+(1-\mu) \lambda \varepsilon]^{S}[(1-\mu) \lambda \varepsilon]^{G}[2(1-\mu)(1-\varepsilon) \lambda]^{N}}
\end{aligned}
$$

When geo-space information is available and with network sharing, the likelihood may be written as:

$$
\operatorname{prob}(G, S, N \mid s=O)=\lambda^{G+S+N}\left[\varepsilon^{G+S}(2(1-\varepsilon))^{N}\right]
$$


Thus from above Eq. (1),(2) and (3), the number of CR entering a particular geo-space and sharing occurs is a sufficient information for a particular reference time $T$. The probability of geo-space available and sharing, geo-space information available and nonsharing and no geo-space information available are assumed using: $(1-\alpha),(\alpha \delta), \alpha(1-\delta)$ and likelihood is

$$
\begin{gathered}
L((G, S) \mid \theta)=(1-\alpha) * e^{-\varepsilon T} \frac{(\varepsilon T)^{G}}{G !} e^{-\varepsilon T} \frac{(\varepsilon T)^{S}}{S !}+ \\
\alpha \delta * e^{-\varepsilon T} \frac{(\varepsilon T)^{G}}{G !} e^{-(\mu+\varepsilon) T} \frac{([(\mu+\varepsilon) T])^{G}}{S !}+\alpha(1-\delta) * \\
e^{-(\mu+\varepsilon) T} \frac{[((\mu+\varepsilon) T)]^{G}}{G !} e^{-\varepsilon T} \frac{(\varepsilon T)^{S}}{S !}
\end{gathered}
$$

$$
L(\alpha, \delta, \mu, \varepsilon)=
$$$$
\prod_{t=1}^{T}\left[(1-\varepsilon)^{N_{t}}(1-\mu)^{N_{t}} A^{G_{t}+S_{t}}\right] \mathrm{X}\left[\alpha ( 1 - \delta ) \left(\frac{\mu}{A}+\right.\right.
$$$$
\left.1)^{G_{t}}+\alpha \delta\left(\frac{\mu}{A}+1\right)^{S_{t}}+(1-\alpha)\left(\frac{1}{1-\mu}\right)^{G_{t}+S_{t}+N_{t}}\right]
$$

where $A=\frac{(1-\mu) \varepsilon}{2}$ and $\mathrm{G}_{\mathrm{t}}$ is the number of sharing for geo-space information available, $\mathrm{S}_{\mathrm{t}}$ as number of nonsharing when geo-space information is available and $\mathrm{N}_{t}$ as number of sharing when geo-space information is not available. For a particular time, the maximum likelihood of sharing parameters $\alpha \mu$ and $\delta$ will be 1 or 0 , which implies a single sharing event occurs with respect to time. The above Fig. 2 shows the likelihood of occurrence of sharing based on the distribution random dynamic $\mathrm{CR}$ transceivers.

Here we have considered one set of Power Spectral Density (PSD) and for some random set of femto-cells or small irregular size cells in different place and at different time. The number of iterations has been taken as 140 and the graph converges at $2.117 \times 10^{\wedge}-6 \mathrm{dBm}$ that is approximately at $1 \mathrm{~mW}$ power and $3.954 \times 10^{\wedge}-7 \mathrm{dBm}$ which is also almost $1 \mathrm{~mW}$. Thus for a large number of data, we can predict the sharing implementation for each day based on proposed model. As the time dimension is taken as independent, the likelihood of sharing data $M=\left(G_{i}, S_{i}\right)_{i=1}^{I}$ over I continuous time is the product of instantaneous likelihood:

$$
L(M \mid \theta)=\prod_{i=1}^{I} L\left(\theta \mid G_{i}, S_{i}\right)
$$

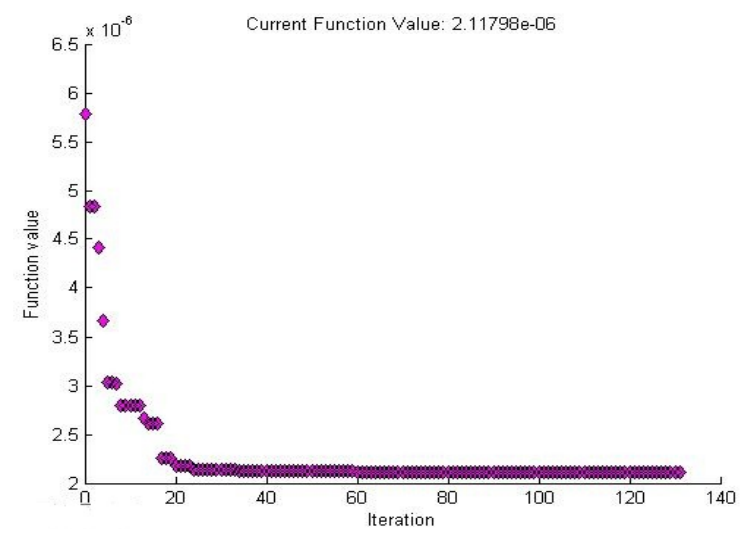

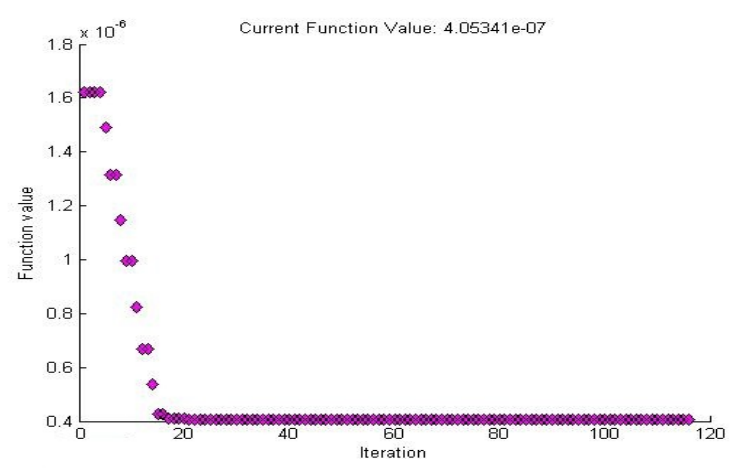

Fig 2.Likelihood of spectrum sharing based on $3 \mathrm{CR}$ transceivers in morning at $0-100 \mathrm{MHz}$ and (a) assuming 50\% informed CR entering a location (b) assuming uninformed CR entering a location

To calculate vector $\theta$ from given $\mathrm{M}$, we maximize the Eq. (6) which provides direct estimates of uninformed and informed CR sharing in a fixed location as well as other parameters effecting the sharing. The Probability of Informed Sharing (PIS) is defined as the unconditional probability of informed CR sharing for a particular time, i.e.

$$
P I S=\frac{\mu_{t}}{\left(1-\mu_{t}\right) \varepsilon_{t}+\mu_{t}}
$$

where, $\left(1-\mu_{t}\right) \varepsilon_{t}+\mu_{t}$ is the probability of performing sharing when the geo-space information is available. The value of PIS is 0.6285 which shows a better feasibility of the model.

\section{Conclusion and discussion}

The paper illustrates the differential behavior of informed and non-informed mobile CR transceivers spectrum sharing activity. Using the designed algorithm, we use geo-space information to formulate the probability of information based sharing for each CR transceiver. It also shows that the chances of non-sharing for informed CR transceivers during its mobility time in a particular location is less as compared with uninformed CR transceiver. This will not only further increase the spectral efficiency but also quality of service using flexible spectrum usage. The work can be further extended by considering the case of adaptive modulation effects on spectrum sharing for the CR transceivers in a mobile condition and the available networks of a particular location or geo-space. 


\section{References}

1. M. Gastpar, On capacity under receive and spatial spectrumsharing constraints. IEEE transactions on Information Theory, 53(2):471-487 (2007)

2. Varaka Uday Kanth Et. al., Spectrum sharing in cognitive radio networks. International journal of engineering trends and technology, 4(4):1172-1175 (2013)

3. X. Kang,Y.C.Liang, H,K,Garg and L.Zhang, Sensing-based spectrum sharing in cognitive radio networks. IEEE Transactions on Vehicular Technology, 58(8):4649-4654 (2009)

4. Stergios Stotas and Arumuga Nallanahan, Enhancing the capacity of spectrum sharing cognitive radio networks. IEEE Transactions on Vehicular Technology, 60(8):3768-3779. (2011)

5. C. Liang, Y. Zeng, E. C. Y. Peh, and A. T. Hoang, Sensingthroughput tradeoff for cognitive radio networks. IEEE Transactions on Wireless Communication, 7(4):1326-1337 (2008)

6. Goldberger and A.S., A course in econometrics, Harvard University Press, Cambridge. (1991)
7. W.H. Hsieh, An experimental study on cam-controlled planetary gear trains. Mechanism and Machine Theory, 42(5):513-525 (2007)

8. W.H. Hsieh and I. C. Lee, Modelling and control of camcontrolled planetary gear trains. International Journal of Modelling, Identification and Control, 12(3):272-279 (2011)

9. H.S. Yan and W.R. Chen, On the output motion characteristics of variable input speed servo-controlled slider-crank mechanisms. Mechanism and Machine Theory, 35(4):541-561 (2000)

10. H. S. Yan, M. C. Tsai, and M. H. Hsu,An experimental study of the effects of cam speeds on cam-follower systems. Mechanism and Machine Theory, 31(4):397-412 (1996)

11. H. S. Yan, A methodology for creative mechanism design. Mechanism and Machine Theory, 27(3):235-242 (1992)

12. C. H. Hsu, A graph representation for the structural synthesis of geared kinematic chains. Journal of the Franklin Institute,330(1):131-143 (1993) 